Classification

Physics Abstracts

42.30

\title{
Analysis and Mapping of Natural Landscapes from Satellite Images Using Morphological Filters
}

\author{
Catherine Mering $\left({ }^{1}\right)$, Yann Callot $\left({ }^{2}\right)$ and Akila Kemmouche $\left({ }^{3}\right)$ \\ $\left({ }^{1}\right)$ Université Pierre et Marie Curie, CNRS URA 1759, T26-16-1E, Boite 129, \\ 4 Place Jussieu, 75005 Paris Cedex, France \\ $\left({ }^{2}\right)$ Université François Rabelais, Département de Géographie, BP 2221, 37021 Tours Cedex, France \\ $\left({ }^{3}\right)$ Université de Sciences et Techonologie Houari Boumédiène, BP 32, \\ Bab-Ezzouar 16111 Alger, Algeria
}

Résumé. - Une approche morphologique est proposée pour étudier la dynamique des paysages naturels à partir d'images satellite. Les milieux étudiés connaissent tous une évolution importante due à des facteurs géologiques ou climatiques. Afin de cartographier les éléments du paysage, témoins de cette dynamique, nous mettons en œuvre des méthodes basées sur les filtres morphologiques.

\begin{abstract}
A morphological approach is proposed for the study of natural landscapes from satellite images. The environment under study is affected by recent evolution due to geological and climatic events. Image processing based on Morphological Filtering is used in order to extract and map the landforms which are the marks of change of the environment.
\end{abstract}

\section{Introduction}

Vegetal and mineral landscapes are continuously transformed during recent periods. Their shapes are modified under the action of internal phenomena such as tectonic and volcanism or external ones, such as climate stress, erosion, or anthropic action. The origins of some landforms may be deduced from their shape: directions and intensity of winds may be deduced from orientation of sand dunes, as main tectonic activity from the morphology of geological structures, intensity of dryness from the discontinuity of vegetation cover in arid lands. Classically, photo interpretation on airborne photographs and nowadays image analysis on spatial image are of crucial help to identify and measure the specific shape of these landforms. The concepts and methods of Mathematical Morphology [1] are used in this work to delimit shape entities that are thematically significant from the grey tone images. 


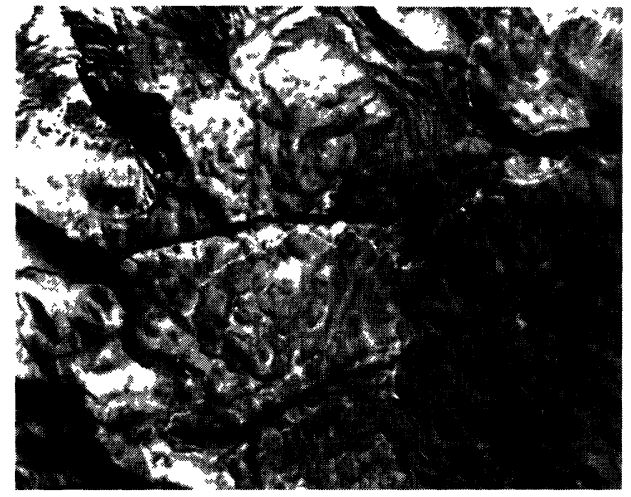

Fig. 1.

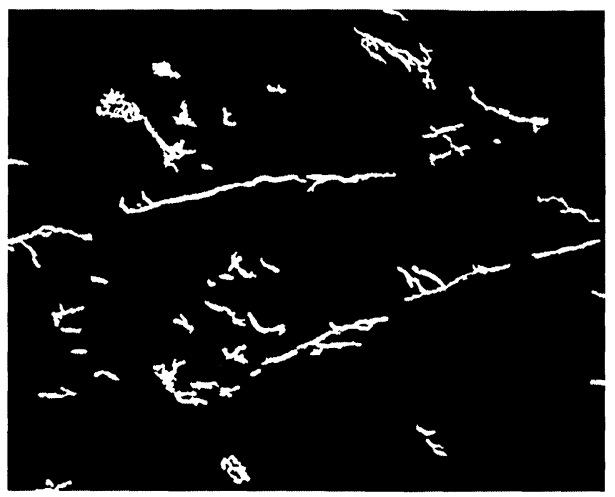

Fig. 2.

Fig. 1. - SPOT Panchromatic subscene on the Hualca-Hualca fault zone $(20 \mathrm{~km} \times 20 \mathrm{~km})$.

Fig. 2. - Extraction of the Hualca-Hualca fault lines.

\section{Fault Mapping in Active Tectonic Zones}

One shows here two types of normal faults analyzed from satellite image on a region of Southern America (Southern Peru and Northern Chile) affected by active tectonic resulting from the subduction of the Nazca Plate under the American continental lithosphere.

The methodology of filtering has been applied to a SPOT subscene $(2000 \times 2000$ pixels $)$ located west of the Hualca-Hualca volcano (Southern Peru) (see Fig. 1). Darkness and thickness of the lines interpreted as faults depend on the geomorphology of the scarps and on the conditions of the viewing. On SPOT images, landforms are detected from the cast shadow resulting from illumination of the relief [2]. In the image of Figure 1, other elements are both linear and dark, but they are not relevant for fault analysis. Consequently, a unique step of filtering is not sufficient to extract significant entities. The transformations that enable the selection of linear objects according to the local contrast as well as to their thickness are top hat transformations. A top hat transformation is defined as the residue of a morphological transformation on a grey-tone function $f$ such as an opening $\gamma$ or a closing $\phi$ with a structuring element $\mathrm{B}$ thresholded at a given level $n$ [3]. A Black Top Hat BTH is the residue of a closing, when a White Top Hat WTH is the residue of an opening:

$$
\mathrm{BTH}=\phi-f ; \quad \mathrm{WTH}=f-\gamma .
$$

One first applies to the image a Black Top Hat in order to extract dark lineaments. The undesired features will be then progressively eliminated after each step of filtering and thresholding by an algorithm called the Geodesic Dilation $(G D)$ [4] which is known as an efficient tool to clean out a binary image by removing undesirable entities. The principle of GD is the following: from a couple of binary images, the first one containing the connected entities to extract but also undesired entities, the second one containing only significant entities even if disconnected, the GD produces a binary image with only the significant entities which have been reconnected. Faults are thin and long connected entities that have to be separated from the other smaller entities according to a size criteria on the binary image, with a geodesic dilation after a binary erosion of size 2 . Steeply dipping faults are sub-rectilinear and have therefore a constant orientation in the image. The pixels having the same Freeman code as the characteristic one of the two faults are selected. 


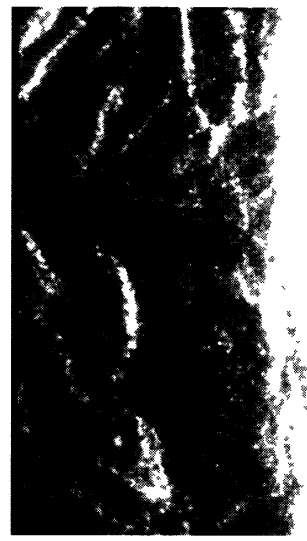

Fig. 3.
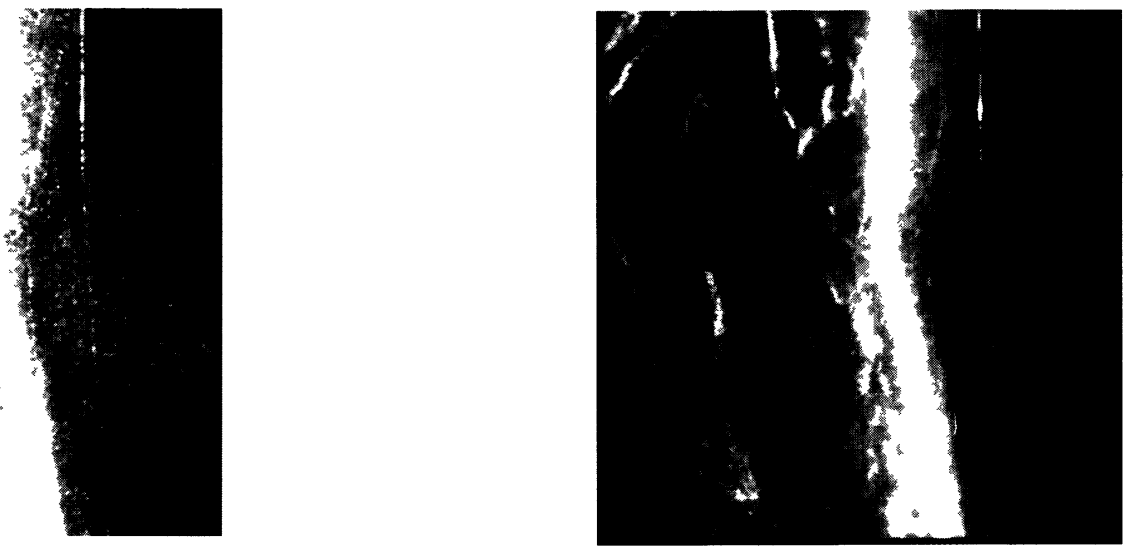

Fig. 4.

Fig. 3. - A detailed view from a SAR-ERS1 scene of the Atacama fault zone.

Fig. 4. - Connected center filter on the image of Figure 3.

A geodesic dilation is performed with these markers. This procedure eliminates rectilinear entities with quite different orientation than that of the faults (Fig. 2).

On radar images such as SAR ERS-1 ones, the slopes of topographic features (facing the antenna) are responsible for strong echoes with the greatest amount of reflection occurring when the local slope is perpendicular to the radar beam. Then, abrupt scarps of recent faults with face radar illumination, can be then considered as creating conditions for such a foreslope brightening. A detail of SAR image of the Atacama fault (Northern Chile) is shown in Figure 3. The features to extract correspond to sharp white continuous lines on the image. It is yet necessary to reduce the speckle noise of the original image. This is achieved here by a connected filter (Fig. 4) which is a built according the same principle than the center filter proposed by Serra [4] to eliminate noise from grey tone images called the connected center filter $\beta_{\mathrm{c}}$ [5]. Given the two filters $\gamma_{\mathrm{c}}$ and $\varphi_{\mathrm{c}}$ which are respectively an opening and a closing by reconstruction, the centre $\beta_{\mathrm{c}}$ between $f_{\mathrm{c}}$ and $g_{\mathrm{c}}$ is defined as follows:

$$
\beta_{\mathrm{c}}=\left(I \wedge f_{\mathrm{c}}\right) \vee g_{\mathrm{c}}
$$

where $f_{c}=\varphi_{c} \gamma_{c} \varphi_{c}$ and $g_{c}=\gamma_{c} \varphi_{c} \gamma_{c}$.

The white lines are then easily extracted from a whole ERS-1 subscene of the fault (Fig. 5) by a high thresholding. The white components still remaining on the image being thinner than the object to extract are eliminated by a geodesic reconstruction, while thicker ones are eliminated after a labelling (Fig. 6).

These techniques are used to detect faults in active tectonic regions from satellite images produced by optical or microwave instruments. Filters based on reconstruction are still time consuming when utilized on entire satellite scenes $(6000 \times 6000$ pixels $)$ and may take hours when implemented with a sequential algorithm, when top hats are processed in few minutes on the same surface.

\section{Mapping of Dunes in Desertic Zones}

The northeastern part of the Great Western Erg (Algerian Sahara) is an area with a continuous cover of coarse sands organized in alignments of large domes separated by corridors where deflation prevails. The aim of the study was here to map automatically deflation zones from SPOT 


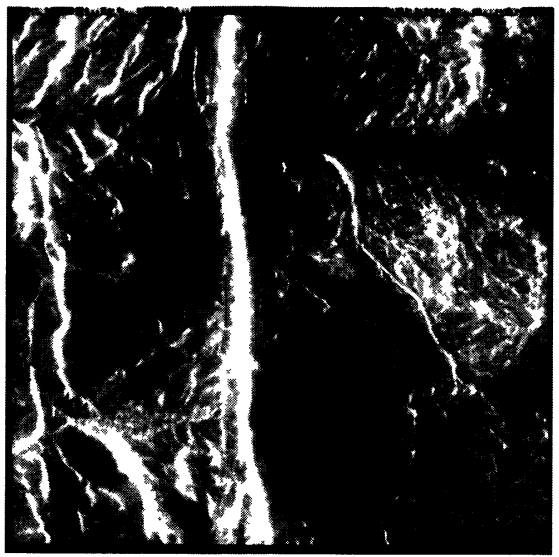

Fig. 5.

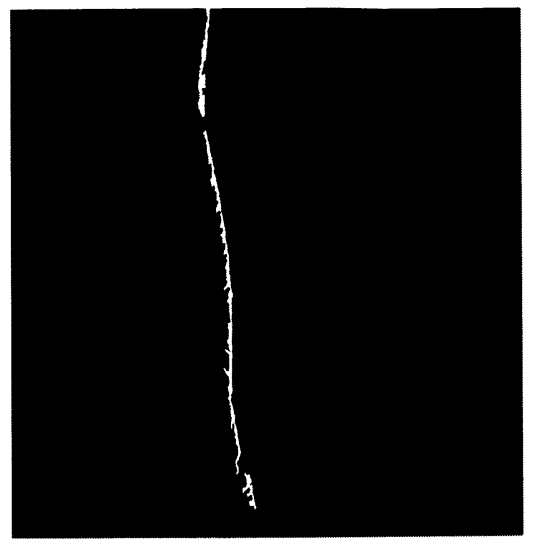

Fig. 6.

Fig. 5. - A whole segment of the Atacama fault on a ERS-1 scene $(25 \mathrm{~km} \times 25 \mathrm{~km})$.

Fig. 6. - Mapping of the Atacama fault line.

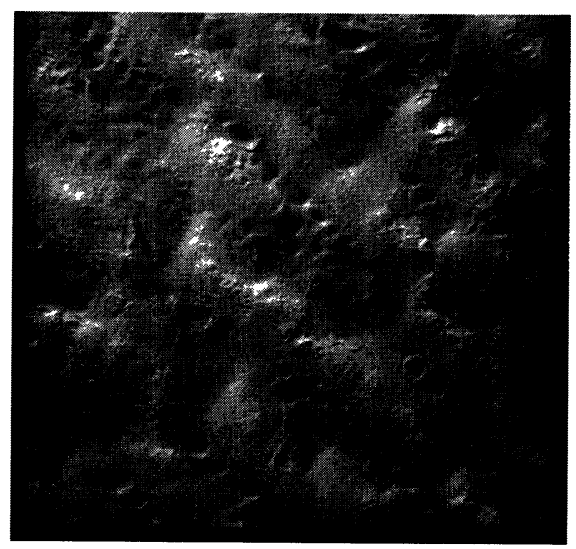

Fig. 7.

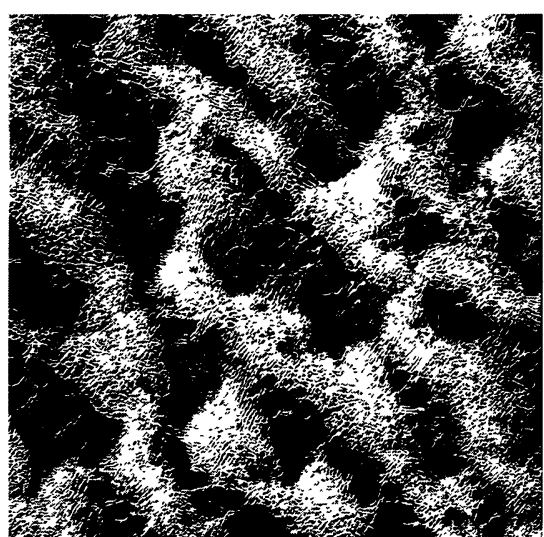

Fig. 8.

Fig. 7. - A SPOT Panchromatic subscene of the Great Western Erg $(10 \mathrm{~km} \times 10 \mathrm{~km})$.

Fig. 8. - Extraction of the seifs by a white top hat.

Panchromatic images (Fig. 7), to know about the direction of the winds responsible of this deflation corridors [6]. The whole mapping is coming from the delimitation of the seifs (small dune shapes). The grey level of their edges facing the sun is very high. Their detection is then possible by the means of a white top hat (Fig. 8). One obtains only the akle (jointed groups of seifs) in the form of stripped areas where the remaining dark zones correspond to the shadowy part of the dunes and to the small sandy interdune areas between the seifs. As this dark interdune areas is narrow enough, it can be eliminated by a dilation of size 2 . The grey tones on the image are sufficiently smoothed and differentiated to enable the delimitation of both sets that one looks for. 


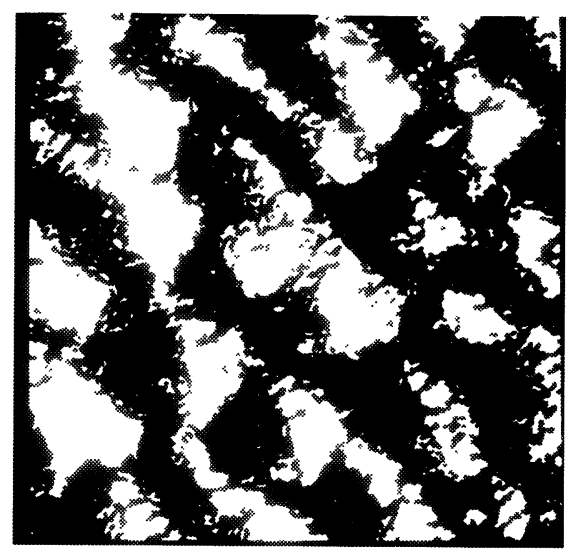

Fig. 9.

Fig. 9. - First extraction of the deflation zones.

Fig. 10. - Mapping of the deflation zones.

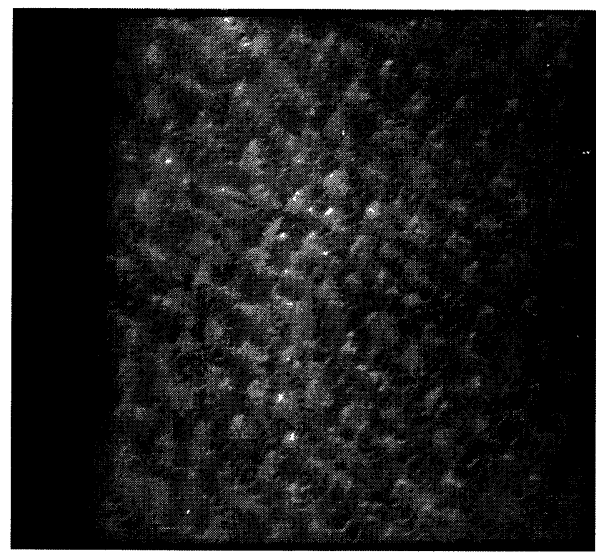

Fig. 11.

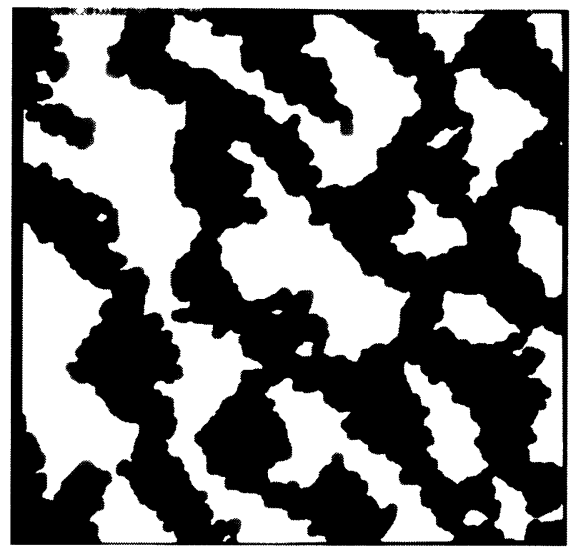

Fig. 10.

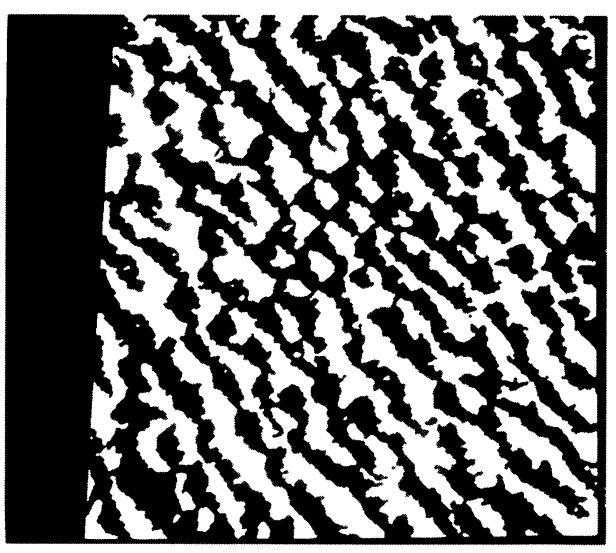

Fig. 12.

Fig. 11. - A whole SPOT subscene of the $\operatorname{Erg}(30 \mathrm{~km} \times 30 \mathrm{~km})$.

Fig. 12. - Mapping of the Erg: the akle zone (in black) and the deflation zone (in white).

The binary image obtained by thresholding with an upper limit corresponds roughly to the set of deflation areas (Fig. 9). A geodesic reconstruction from a marker obtained by a binary erosion of size 2 eliminates the scattering of small isolated entities since they should not be identified to deflation areas, but to interdune zones in the akle areas. The small holes remaining inside large deflation areas are eliminated by the same transformation on the complementary set. The final image (Fig. 10) obtained by this way corresponds to the deflation areas. The sequence of morphological transformations described above may be rapidly implemented on large zones in Figure 11, where a SPOT subscene which corresponds to a square of $30 \mathrm{~km}$ on the ground is shown. The resulting map (Fig. 12) allows one to distinguish akle areas (in black), with fine and unstable sands, 


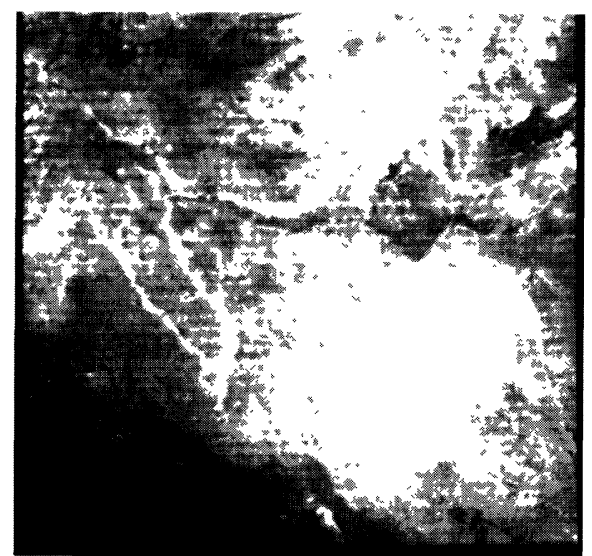

Fig. 13.

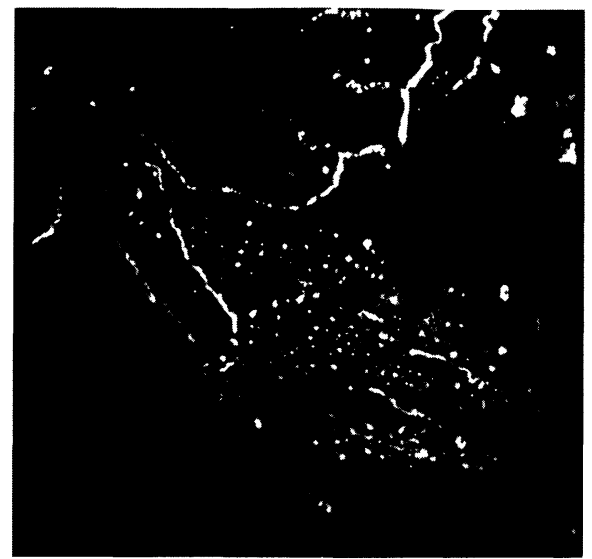

Fig. 14.

Fig. 13. - A vegetation index computed from a Thematic Mapper subscene of the Lagouat region (24 km on $24 \mathrm{~km})$.

Fig. 14. - A vegetation map obtained from a high thresholding of the image of Figure 13.

which are an immediate danger for the environment, from more stable areas (in white) that are less dangerous at present, but still liable to deteriorate.

\section{Mapping of Vegetation Density in Arid Lands}

In arid and semi arid lands, ligneous vegetation cover tends to contract and concentrate within small topographic depressions under climatic stress and anthropic action. Each of the various types of its spatial organization are known as different steps towards desertification. In this context, maps of vegetation density are more relevant than classical vegetation maps to estimate the stage of desertification on large areas. The idea was here to obtain these maps according to the principles of granulometric analysis on images [7]. The size parameter utilized is derived from the granulometric density, $g(\lambda)$, which gives the proportion of area suppressed between two successive openings by convex structuring elements of increasing size such as $\mathrm{B}(\lambda)$ and $\mathrm{B}(\lambda+1)$. On the Thematic Mapper scene of the Lagouat region (North of the Algerian Sahara), a vegetation index is computed from original TM bands (Fig. 13) and thresholded (in white in Fig. 14). One can see the presence of vegetation inside the dayas (small and dispersed spots) and the oueds (large linear bands). The granulometric density is computed from a sliding window centered on each pixel of the binary image, with a square structuring element. The choice for the size of the sliding window has been done according to the distance between two peaks of the horizontal covariogram [1] computed from the whole binary image (Fig. 14). The optimal size for the sliding window was then 100 pixels (that is 3 kilometers on the ground). By this way, each pixel is described by $n$ parameters $g(n)$ corresponding to the value of the granulometric density obtained at step $n$. The grey tone image of $g(1)$ is shown in Figure 15. The pixels are then classified according to these $N$ variables $g(n)$, by a $k$-means classification. The result obtained with 7 classes is presented in Figure 16. One can see in white, the parts of the scene where there is the highest density of vegetation, as the black parts correspond to a lack of vegetation. The five other grey tones correspond to heterogeneous textures, each class being composed of particles of each size, from the smallest 


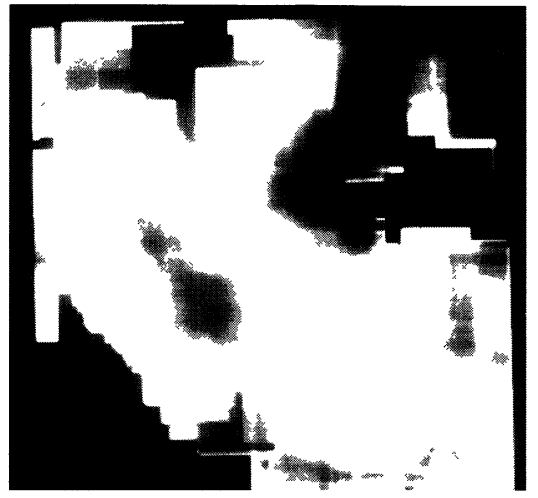

Fig. 15.

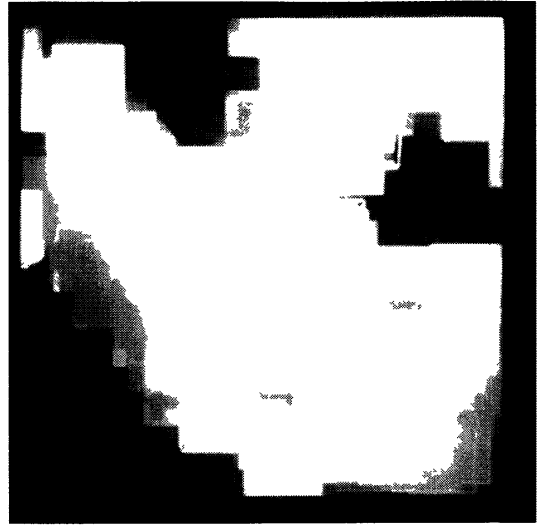

Fig. 16.

Fig. 15. - The grey-tone image of $g(1)$ computed from the image of Figure 14.

Fig. 16. - The final map of vegetation density.

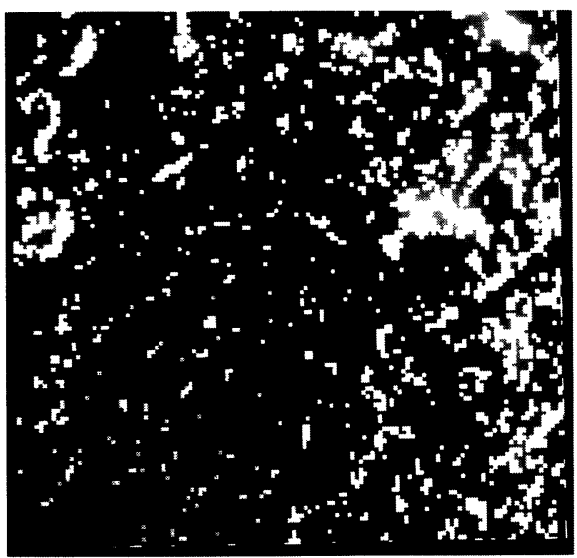

Fig. 17.

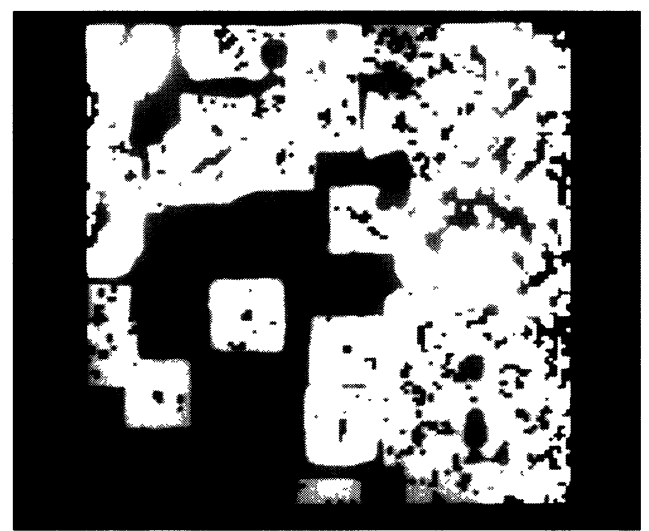

Fig. 18.

Fig. 17. - Thresholding of the vegetation index NDVI from a SPOT scene of Bidi $(10 \mathrm{~km} \times 10 \mathrm{~km})$.

Fig. 18. - The final map of vegetation density of Bidi (in grey) surimposed with the vegetation map (in black).

ones (size one) to the biggest (size three), with an increasing proportion of big spots according to the number of the class.

Another experiment has been done from an extraction of a SPOT image of the region of Bidi (Northern part of Burkina Faso) in the Sahel. As the variation of the size of the spots of vegetation were not so big, we zoomed three times the binary image obtained from a thresholded Normalized Vegetation Index (NDVI) (Fig. 17). The result of the whole process is presented in Figure 18, where the original image (in black) is surimposed over the final map (in grey tones).

In arid regions, ligneous vegetation is often the only resource for breeding and heating for rural societies. One of the aim of these mapping techniques is then to survey the state of ligneous resources from repetitive observations given by satellite images. 


\section{References}

[1] Serra J., Image Analysis and Mathematical Morphology, Vol. 1 (London, Academic Press, 1982).

[2] Mering C., Huaman D., Chorowicz J., Deffontaines B. and Guilande R., New Data on the geodynamics of southern Peru from computerized analysis of SPOT and ERS1 images, Tectonophysics 259 (1996) 153-170.

[3] Meyer F., Contrast feature extraction, Pract. Met. 8 (1978) 374-380.

[4] Serra J., Image Analysis and Mathematical Morphology, Vol. 2 (London, Academic Press, 1988).

[5] Mering C. and Parrot J.F., Radar Image Analysis using Morphological Filters, in "Mathematical Morphology and its applications to Image Processing", P. Soille, Ed. (Kluwer Academic Publ., 1994) pp. 353-360.

[6] Callot Y., Mering C. and Simonin A., Analysis of sand hills massifs on high resolution images: an application to the Great Western Erg (Algeria), Int. J. Remote Sensing 15 (1994) 3799-3822.

[7] Matheron G., Random Sets and Integral Geometry (Wiley, New York, 1975). 